\title{
Genetics of Drosophila simulans male mating discrimination in crosses with $D$. melanogaster
}

\author{
MC Carracedo, A Asenjo and P Casares \\ Area de Genética, Departamento de Biología Funcional, 33071 Oviedo, Asturias, Spain
}

The genetic bases of sexual isolation between Drosophila melanogaster and $D$. simulans have been mainly studied in females, and there is little information about the role of the males in interspecific mating discrimination. Using D. simulans synthetic lines with compound chromosomes from a population of the Seychelles Islands (high frequency of interspecific mating) and a multimarker strain (low frequency), we show that $D$. simulans males play an important role in discriminating $D$. melanogaster females.
The genetics of male discrimination fits well with the inheritance mode of a single locus, dominant for sexual isolation, located in chromosome II near the net mutation (2L-0.0). The heterospecific mating success of the male was not related to his sexual vigor. The specific load of male cuticular hydrocarbons was counted as a possible source of discrimination used by the $D$. melanogaster female.

Heredity (2003) 91, 202-207. doi:10.1038/sj.hdy.6800328

Keywords: Drosophila simulans; D. melanogaster, male interspecific sexual discrimination

\section{Introduction}

Sexual isolation between related species is a consequence of genetic changes occurring in both sexes that accumulate in reproductively isolated populations during the speciation process. In animals, such changes are related to differences in courtship behavior and to signals used in mate recognition, which cause the avoidance of interspecific mating. The most important kinds of signals used by the two sexes in mating recognition are visual, acoustic and chemical (Kawanishi and Watanabe, 1981; Kyriacou and Hall, 1982; Coyne et al, 1994; Coyne and Oyama, 1995)

Knowledge of the number, location, mode of inheritance and effects of 'speciation genes' involved in reproductive isolation is the main focus of the genetics of speciation. To study these genes, the first step is to detect them, which can only be carried out when inter- or intrapopulational variability for isolation is present.

The occurrence of variability in the degree of sexual isolation among related species of Drosophila is often seen in laboratory crosses. In the pair of sibling species Drosophila melanogaster and D. simulans, interspecific crosses occur easily in the laboratory, especially when the females are $D$. melanogaster. Inter- and intrapopulational studies have repeatedly shown differences in the frequency of interspecific matings, associated with different behaviors in both the males and the females, which indicates genetic variance in both sexes for the degree of sexual isolation (Watanabe et al, 1977; Carracedo and Casares, 1985; Carracedo et al, 1991, 2000b; Das et al, 1995). Despite the involvement of both sexes in sexual isolation, most genetic studies using this

Correspondence: M Carracedo, Area de Genética, Departamento de Biología Funcional, 33071 Oviedo, Asturias, Spain.

E-mail:mcc@correo.uniovi.es

Received 21 April 2002 pair of sibling species have been carried out in females (Coyne, 1989, 1992; Carracedo et al, 1989, 1995, 1998), probably because this sex is considered as playing the more important role in recognizing, and accepting or refecting, homo- and interspecific mating (Cowling and Burnet, 1981).

As pointed out above, males of $D$. simulans are known to play an important role in interspecific mating with D. melanogaster females, since (i) genetic variation for mating by males has been detected (Watanabe et al, 1977; Carracedo and Casares, 1985; Carracedo et al, 2000b) and (ii) there is a response to artificial selection for increasing heterospecific matings (Eoff, 1977; Izquierdo et al, 1992). Which male characteristics are involved in a greater or lesser heterospecific mating frequency is, however, not known. Carracedo et al (2000b) searched for a relationship between homospecific mating success and the frequency of heterospecific mating, without success, and Jamart et al (1993) also failed to relate male mating propensities with the frequency of hybridization.

Research during the last few years has stressed the role of cuticular compounds with pheromonal activities in the level of sexual isolation between related species of the melanogaster group (Antony and Jallon, 1982; Antony et al, 1985; Coyne et al, 1994; Coyne and Oyama, 1995; Savarit et al, 1999). If a D. melanogaster female is able to recognize and accept a male in accordance with his pheromonal profile, then male variation in the amount and type of his cuticular compounds could determine male success in heterospecific mating.

In this regard, Scott (1994) found that D. melanogaster females carried genes in chromosome III for the discrimination between males in accordance with their cuticular hydrocarbons, although Cobb and Ferveur (1996) 'consider that his data do not necessarily justify his conclusions' and that differences among male mating success may be explained by other male behaviors involved in female stimulation. 
The study of the genetics of sexual isolation between $D$. melanogaster and D. simulans is difficult, because progeny are sterile and it is not possible to perform introgression of phenotypic, biochemical or molecular markers between the chromosomes of the two species, as is usually performed with related species having at least one fertile hybrid sex (Coyne, 1989, 1992). This problem can be overcome in part by using synthetic lines built from strains with high and low levels of interspecific mating. The present paper uses this method in an attempt to gain knowledge of the role of male cuticular pheromones in male heterospecific mating success. We used two male $D$. simulans populations characterized by having (i) a very different success in mating with D. melanogaster females, and (ii) a different composition of cuticular hydrocarbons. Synthetic lines carrying chromosomes with different fragments of the two populations were produced following the methodology of Carracedo et al (2000a). These lines were examined for sexual isolation and for their male cuticular compound profile.

\section{Materials and methods}

Fly stocks and culture conditions

A wild D. simulans strain from the Seychelles Islands whose males hybridized around $60 \%$ with the females of $D$. melanogaster (preliminary results) was used for the study. This $D$. simulans strain was kindly sent to us by JR David in 1988.

A D. simulans stock homozygous for several recessive markers: forked-2 (short bristles) on the $X$ chromosome ( $\left.f^{2}: 1-56.7\right)$; net (altered wing veins) and brown (eye color) on the two arms of the second chromosome (nt: 2-0.0, 2L; bw: 2-104.5, 2R); and scarlet (eye color) and ebony (black body color) on the two arms of the third chromosome (st: 3-40.0, 3R; e: 3-60.0, 3L). Mutations brown and scarlet in the same individual give a peach-colored eye. Map locations for $f^{2}, n t$ and $b w$ are taken from $D$. melanogaster, whose $X$ and second chromosomes are homosequential to those of D. simulans (Lemeunier and Ashburner, 1976, 1984). Locations of $s t$ and $e$ correspond to those identified directly in D. simulans (Ohnishi and Voelker, 1979). The tiny fourth chromosome was not studied because markers were not available. This stock was derived from the Bloomington Stock Center (stock no. 2008).

A D. melanogaster wild-type population captured in Asturias (Spain) in 1998, named Mellan, was used. Females of this population were used for interspecific mating estimates.

The flies were reared on a medium made up of agar $(1.2 \%)$, baker's yeast $(20 \%)$, sugar $(10 \%)$, salt $(0.2 \%)$ and propionic acid $(5 \mathrm{ml} / \mathrm{l})$, and under a cycle of $12: 12 \mathrm{~h}$ of light:darkness in a climatic chamber. Fly culture and all experimental tests were conducted at $21^{\circ} \mathrm{C}$.

\section{Construction of synthetic lines}

Crosses between the multimarker stock and the Seychelles strain were achieved to obtain lines with chromosomes X, II and III composed of different fragments from both genomes. Figure 1 outlines the two reciprocal crosses (A and $B$ ) carried out to construct lines that differ in the $\mathrm{X}$ and $\mathrm{Y}$ chromosomes origin.
Six synthetic lines were constructed following a procedure similar to that outlined in Figure 1 for the net brown line. Males and females from the $F_{1}$ of cross $B$ were crossed, and from the different phenotypes that segregated in the $\mathrm{F}_{2}$, we selected males homozygous for net and brown. Each of these was backcrossed (i) to Seychelles females (one male and five females per vial). Males and females that emerged from each vial were left to mate freely (ii) and, from their progeny, we selected net, brown males, which were again individually crossed with five Seychelles females (i). Crosses (ii) and (i) were carried out eight times to obtain finally males with cytoplasm, X, Y and III chromosomes from Seychelles, except for the net to brown fragment in the second chromosome from the multimarker strain.

To confirm that the eight backcrosses were effective in removing the heterozygous markers present in cross (ii), several males from each line were crossed to multimarked females. In all cases, the phenotypes of their progenies matched well with those expected, indicating that the synthetic lines only carried the selected markers.

\section{Intraspecific male mating success}

To assess if the different mutations used could differently affect the male mating success of their carriers, we registered the number of matings out of 100 of the different lines with standard females from Seychelles. To do so, one male from the nt line, for example, was put with one female from Seychelles (both sexes were virgins and aged for 3 days) and observed for $30 \mathrm{~min}$. A total of 100 pairs were scored. The same procedure was followed for studying the male mating success of the $b w, s t, e$ and 2008 lines, and the Seychelles population.

\section{Interspecific male mating propensity}

The level of interspecific male mating propensities accomplished by $D$. simulans was estimated by the number of fertilized D. melanogaster females in 'no choice' tests. Single pairs of D. simulans males and $D$. melanogaster females, newly emerged, were put into vials with food. After 10 days, vials were examined for the presence of hybrid larvae. This method is equivalent to searching for heterospecific sperm in the spermathecae and seminal receptacles of dissected females (Carracedo et al, 1998). Around 100 males were analyzed for each chromosomal class. The experiments were carried out in two blocks of 50 males each.

\section{Hydrocarbon extraction and gas chromatography}

Extraction of flies' cuticular compounds was performed following the procedure of Scott and Jackson (1988). A total of 10 males of a given line, virgin and aged for 4-5 days, were anesthetized, transferred to an empty glass microvial and soaked in $100 \mu \mathrm{l}$ of $\mathrm{n}$-hexane for $2 \mathrm{~min}$. The hexane carried $10 \mathrm{ng}$ of eicosane to serve as an internal standard. Gentle agitation was made every $60 \mathrm{~s}$. Then the solvent was drawn to a new vial and concentrated under a flow of helium.

A volume of $2 \mu \mathrm{l}$ of this extract was injected into an HP 9600 gas chromatograph equipped with a $30 \mathrm{~m}$ DB1 capillary column. During the first ramp, the column temperature increased from 190 to $240^{\circ} \mathrm{C}$ at a rate of $2.5^{\circ} \mathrm{C} / \mathrm{min}$. The second ramp attained $290^{\circ}$ at a rate of 
CROSS A

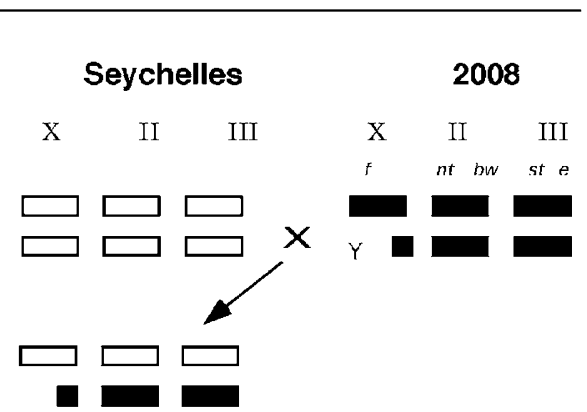

CROSS B

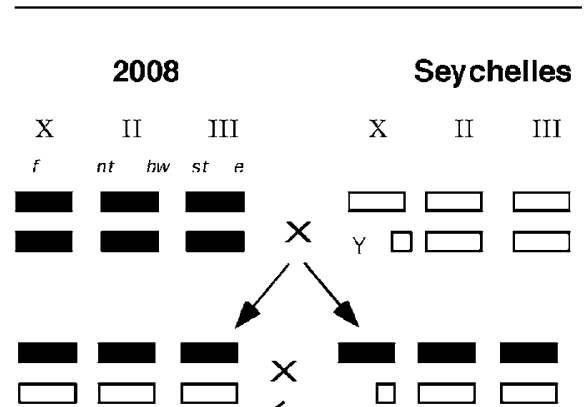

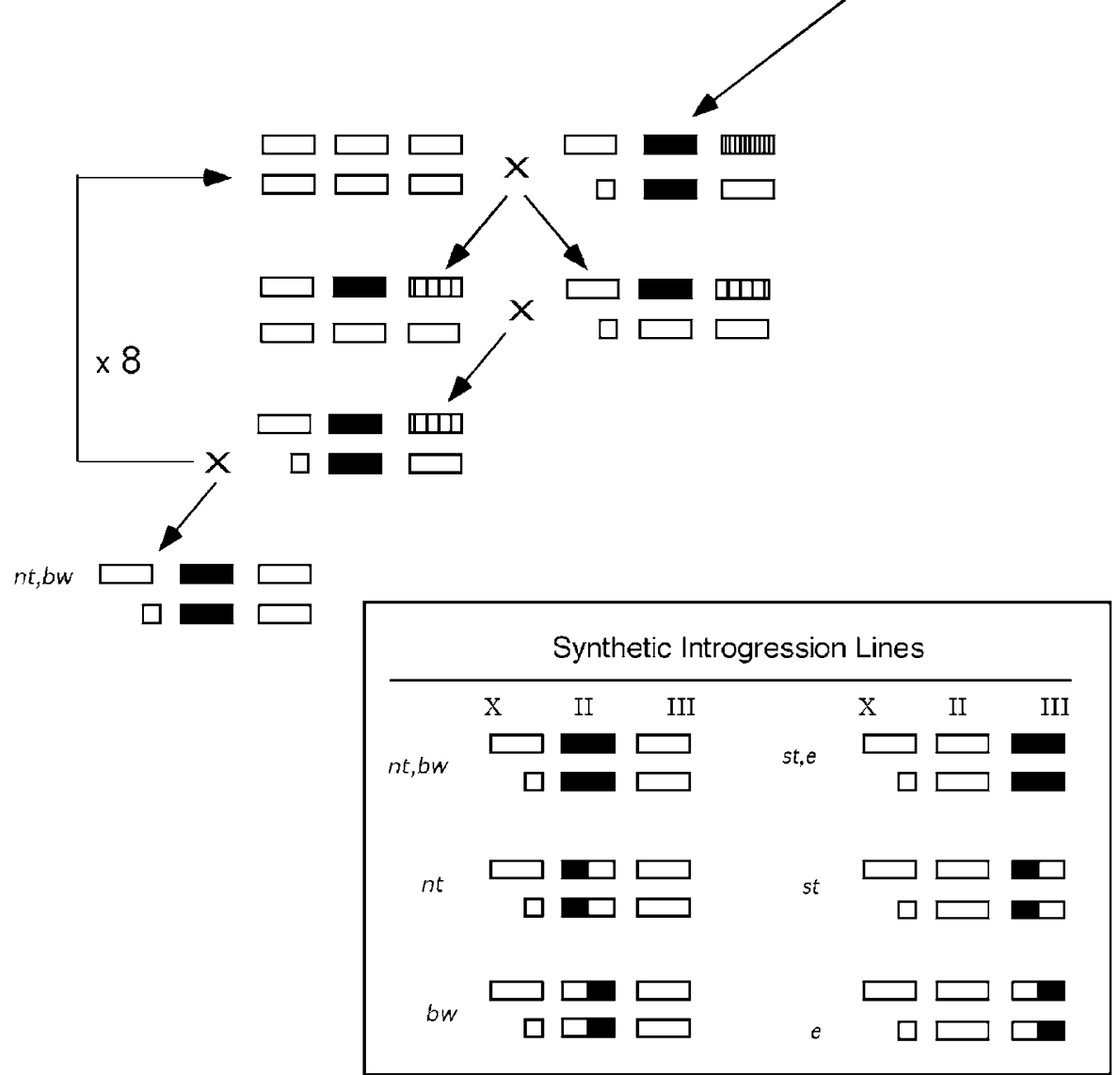

Figure 1 Scheme of the crosses carried out to obtain the $n t, b w$ synthetic introgression line and representation of six lines with different II and III chromosomes.

$8^{\circ} \mathrm{C} / \mathrm{min}$. For each line, three replicates of 10 males each were analyzed.

Hydrocarbon peaks were identified by comparing their comigration with known standards and with published $D$. simulans profiles. The relative amount of each compound per line was calculated by comparing its peak area with that of eicosane.

\section{Results}

Intraspecific male mating success

The percentages of males from the different lines mating in $30 \mathrm{~m}$ with conspecific females were $65 \%$ (2008), $86 \%$ (Seychelles), 82\% (nt), 92\% (bw), 85\% (st) and 80\% (e). A test of variance for homogeneity of binomial distributions (Snedecor and Cochran, 1967) indicates differences between them $\left(\chi^{2}=26.56, \mathrm{df}=5, P<0.001\right)$. The lowest value corresponds to the multimarker strain 2008, whereas there are no differences among the other lines. We conclude that the single mutations used here have no effect on male sexual ability or that any effect is small.

Interspecific mating: effects of $X$ and $Y$ chromosomes Interspecific mating of Seychelles males was very high $(67 \%, 71 / 106)$ in comparison with that of the 2008 strain $(12 \%, 14 / 125)$. The values in $F_{1}$ males from reciprocal crosses A and B were 12.5\% (12/96) and $11 \%$ (9/82), respectively. The difference between these two percentages is not significant in a contingency ' $\chi^{2 \prime}\left(\chi^{2}=0.1\right.$, $\mathrm{df}=1, P=0.75)$, a result that suggests no effect of cytoplasm or $\mathrm{X}$ and $\mathrm{Y}$ chromosomes in sexual isolation. 
The two $F_{1}$ values were pooled in a new value (21/178; $11.79 \%$ ) that differs from the Seychelles strain but not from the 2008 line, suggesting that the male interspecific success behaves mainly as being dominant for sexual isolation (low interspecific mating).

\section{Interspecific mating: effects of second and third chromosomes}

The frequencies of males of the six synthetic lines, Seychelles and the 2008 strain that mated interspecifically, are shown in Figure 3. The two replicates appear pooled. To test the effect of chromosomes II and III on sexual isolation, the values of Seychelles, 2008, and the two $s t, e$ and $n t, b w$ lines were analyzed for independence in a two-way table on the log-linear scale (Bishop et al, 1975). We follow a hierarchical procedure starting with a linear model in which main effects and interactions are present: the value in a cell is the sum of a mean value, factors $\mathrm{A}$ and $\mathrm{B}$, and the $\mathrm{AB}$ interaction term. The analysis first adjusts the observed data to this model, and then searches for the simplest model that fits the data well.

Results of the analysis indicate that chromosome II has a large effect on isolation (G-statistic 75.3 with $1 \mathrm{df}$; $P<0.001)$ with its contribution to the observed data being $93 \%$, while chromosome III effect is weak $(\mathrm{G}=4.8$, 1 df; $P=0.028$ ) contributing $7 \%$ only. No chromosome interaction was detected.

The importance of each arm of chromosome II was examined in the same manner, using the four lines with the same chromosome III but with different arm composition for chromosome II. No arm interaction was detected, so the fitted linear model only includes main effects. The left arm (IIL) has a great effect on isolation $(\mathrm{G}=68.3,1 \mathrm{df} ; P<0.001)$ that can be estimated as $88 \%$ of the total, while the right arm has a small, hardly significant effect on this trait $(\mathrm{G}=4.31,1 \mathrm{df}$; $P=0.038$ ). This result is easily observed from Figure 2,

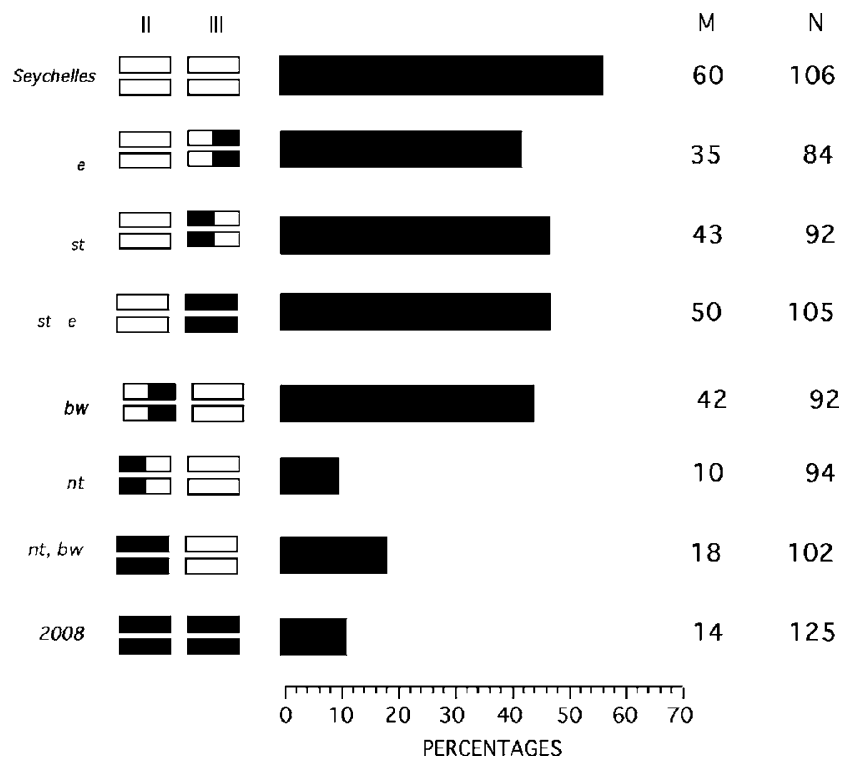

Figure 2 Number of males $(M)$ of each line that achieve interspecific mating. $N=$ number of tested males. where it can be seen that the presence of the net mutant in IIL splits the eight lines into two well-defined groups, one with mating frequencies around $50 \%$ and the other with frequencies around $12 \%$. This is statistically confirmed by an unplanned homogeneity test of percentages (Sokal and Rohlf, 1995), which gives a highly significant result $\left(\chi^{2}=120.7, \mathrm{df}=7, P<0.001\right)$ and allows us to group strains into two groups that show a significant difference:

Sey st st,e e bw nt,bw nt 2008

Those strains joined by a horizontal line do not differ at the $5 \%$ level.

We conclude that a gene/s located near the mutation net (2L-0.0) is involved in the large difference in male sexual isolation detected between the Seychelles and 2008 strains of D. simulans.

\section{Cuticular hydrocarbons}

Table 1 shows the peaks identified from the chromatograms and their relative amount of hydrocarbons in males from the $n t, b w, e$ and st synthetic lines, and the 2008 and Seychelles strains. Peaks that did not reach the relative frequency of $3 \%$ in all lines were excluded from the analysis.

The last row in Table 1 shows the percentages of interspecific mating (from Figure 3) achieved for the different lines.

Since the four synthetic lines examined have their $X$ chromosome from the Seychelles strain, they cannot be compared with the 2008 strain, but can be compared with Seychelles. Thus, any difference between Seychelles and a given line can be imputed to the presence of an arm of a chromosome from 2008. In this way, we have compared, for every peak, the amounts found in Seychelles and in the four synthetic lines by one-way ANOVA. Differences were significant only for the branched pentacosane, heptacosane and nonacosane. Of these, the most important is heptacosane as it represents around $12 \%$ of total hydrocarbons. It is interesting to note the lack of between-line differences in 7-tricosene, the main compound in males of $D$. simulans, which represents more than $50 \%$ of the extracted cuticular compounds.

In an attempt to find a possible relationship between the amount of a cuticular compound and the frequency of interspecific mating, we calculated correlations between these two traits for the peaks that, as detailed above, showed significant between-line differences. The percentages of interspecific mating were subjected to the arc-sine transformation prior to analysis in the parametric scale (Sokal and Rohlf, 1995). The Pearson's correlation coefficients for data in Table 1 were $0.53(P=0.27), 0.70(P=0.12)$ and $0.59(P=0.21)$ for the branched pentacosene, heptacosane and nonacosane peaks, respectively. This absence of a relationship between cuticular hydrocarbons and sexual isolation is graphically shown in Figure 3. Whereas the presence of the net mutation causes a great difference in sexual isolation, it is not accompanied by a parallel change in the cuticular compound profile. Therefore, no relationship is found between cuticular hydrocarbons and interspecific mating in the $D$. simulans males. 
Table 1 Relative mean values (ng) of different cuticular hydrocarbons found in males from synthetic lines and strains

\begin{tabular}{|c|c|c|c|c|c|c|}
\hline Peaks & 2008 & Seychelles & net & brown & ebony & scarlet \\
\hline Branched tricosane & $13.55 \pm 2.87$ & $8.35 \pm 2.61$ & $13.07 \pm 0.91$ & $13.14 \pm 0.97$ & $6.46 \pm 0.21$ & $9.05 \pm 2.77$ \\
\hline 7-Tricosene & $157.24 \pm 12.24$ & $72.72 \pm 7.23$ & $95.31 \pm 5.29$ & $95.82 \pm 4.68$ & $64.71 \pm 8.48$ & $92.36 \pm 12.36$ \\
\hline$n$-Tricosane & $35.71 \pm 3.45$ & $13.43 \pm 2.25$ & $18.06 \pm 1.79$ & $17.91 \pm 2.10$ & $13.18 \pm 2.21$ & $17.68 \pm 2.42$ \\
\hline Tetracosane & $5.18 \pm 0.57$ & $3.47 \pm 0.53$ & $3.53 \pm 0.57$ & $3.52 \pm 0.52$ & $3.19 \pm 1.03$ & $4.19 \pm 0.25$ \\
\hline Branched pentacosane & $5.89 \pm 1.10$ & $2.72 \pm 0.37$ & $7.03 \pm 0.09$ & $7.08 \pm 0.07$ & $0.81 \pm 0.42$ & $0.21 \pm 0.01$ \\
\hline 7-Pentacosene & $9.00 \pm 1.76$ & $6.75 \pm 1.26$ & $12.31 \pm 2.62$ & $11.81 \pm 1.46$ & $6.69 \pm 0.69$ & $10.98 \pm 1.65$ \\
\hline Heptsacosane & $19.66 \pm 2.42$ & $14.52 \pm 1.26$ & $20.63 \pm 1.42$ & $19.28 \pm 2.01$ & $12.15 \pm 1.37$ & $15.59 \pm 2.51$ \\
\hline Nonacosane & $15.56 \pm 1.62$ & $3.10 \pm 0.38$ & $3.71 \pm 0.60$ & $4.20 \pm 0.51$ & $6.24 \pm 0.45$ & $6.81 \pm 0.86$ \\
\hline$\%$ Hybridization & 11.20 & 56.60 & 10.64 & 45.65 & 41.67 & 46.74 \\
\hline
\end{tabular}

The last row shows the hybridization percentages of the lines with D. melanogaster females.

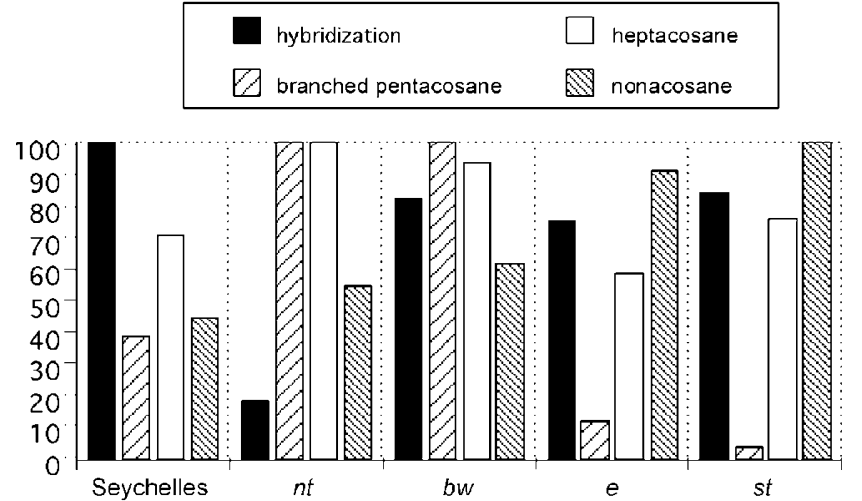

Figure 3 Relative frequency of hybridization and amounts of three male cuticular hydrocarbons found in Seychelles and synthetic lines. Data have been taken from Table 1 . All values are relative to 100 , which is the value assigned to the line with the highest value in Table 1.

\section{Discussion}

Accepting the idea that mating is the final expression of a specific mate recognition system (MRS), sexual isolation results when the MRS of individuals from different populations or species are insufficient, during recognition and courtship, to finally achieve copulation. Therefore, genetic studies of sexual isolation between related species may reveal, for a given species, the genetic systems most directly related to this failure to mate interspecifically. These genes are commonly called discrimination genes, but it is necessary to remember that they are not genes that function to discriminate against foreign individuals, but genes that cause isolation from individuals that do not share all elements of the species' own MRS.

The deciphering of causes affecting interspecific mating is difficult since, during mating, there are two sexes that interchange a battery of different stimuli, the emission and reception of each being linked to different genetic and physiological systems. Thus, when we examine males and detect a gene involved in sexual isolation, we do not know if we are dealing with the ability of these males to achieve interspecific mating through a behavioral change, or if the effect could be ascribed to variation in the male cuticular pheromones detected by females. From the strains and lines analyzed in this study, we have detected a gene/s located on the left arm of chromosome II that affects the male frequency of interspecific mating. However, we do not find a relationship between this change in sexual isolation and the cuticular hydrocarbon composition of the males, which suggests that the detected gene directly affects the male's ability to mate interspecifically.

Our study reveals two important genetic aspects. Firstly, the autosomal genetic system implicated in the interspecific mating of the $D$. simulans male is located on the second chromosome, probably near the telomeric region, since it mapped close to the net mutation (2L-0.0). Secondly, the inheritance mode of this genetic system is dominant for high sexual isolation, and is not affected by either the $\mathrm{X}$ and $\mathrm{Y}$ chromosomes or by maternal cytoplasmic effects.

A characteristic problem associated with the discovery of genes that affect complex traits is to draw conclusions about their effects and frequency. Our results support the occurrence of a single gene or block of genes explaining the great isolation difference that is detected between the Seychelles population and the 2008 strain. However, given the complexity of mating, it would not be a surprise to find that different populations have different genes affecting the same type of sexual isolation. For instance, and unlike our assumption of a single factor, there could be many genes with additive effects for isolation between the same two species, as was inferred from the slow response to artificial selection for increasing sexual isolation found in two independent studies (Eoff, 1977; Izquierdo et al, 1992).

If we accept that sexual isolation between related species reveals the lack of fit between the SMRs of individuals of different sexes and species, the genes detected from isolation studies are those most directly implicated in the auditive, visual, olfactory, tactile and behavioral elements that differentiate the particular populations chosen for the study. If between two $D$. melanogaster populations, there are at least seven genes involved in an incipient sexual isolation (Ting et al, 2001), it is logical to assume that these same seven genes also participate, in addition to many others, in the $D$. melanogaster vs $D$. simulans isolation.

There is no doubt that population differences have appeared after the initial process that created two species. Therefore, the appearance of genetic variants affecting isolation should be observed as specific events that occurred during the evolutionary history of each 
population (Templeton, 1982; Carracedo et al, 1998). Thus using the term 'speciation genes' for genes discovered in particular populations and under particular methodologies seems to be unrealistic.

\section{Acknowledgements}

We acknowledge the Bloomington Stock Center for supplying the 2008 D. simulans strain. This work was supported by the Ministry of Education and Culture of Spain (DGICYT Grant DGE-98 PB 97-1277).

\section{References}

Antony C, Davis TL, Carlson DA, Pechiné JM, Jallon JM (1985). Compared behavioral responses of male Drosophila melanogaster to natural and synthetic aphrodisiacs. J Chem Evol 11: $1617-1629$

Antony C, Jallon JM (1982). The chemical basis for sex recognition in Drosophila melanogaster. J Insect Physiol 28: 873-880.

Bishop YM, Fienberg SE, Holland PW (1975). Discrete Multivariate Analysis: Theory and Practice. MIT Press: Cambridge, MA.

Carracedo MC, Asenjo A, Casares P (2000a). Location of Shfr, a new gene that rescues hybrid female viability in crosses between Drosophila simulans females and D. melanogaster males. Heredity 84: 630-638.

Carracedo MC, Casares P (1985). Intrapopulation genetic variation in the hybridization between Drosophila melanogaster females and D. simulans males. Experientia 41: 106-108.

Carracedo MC, Casares P, Izquierdo JI, Piñeiro R (1991). Receptivity and sexual maturation of Drosophila melanogaster females in relation to hybridization with $D$. simulans males: a populational analysis. Anim Behav 42: 201-208.

Carracedo MC, García-Flórez L, San Miguel E (1989). Sexual maturation in Drosophila melanogaster females and hybridization with $D$. simulans males: a study of inheritance modes. J Hered 80: 157-158.

Carracedo MC, Piñeiro R, Casares P (1995). Chromosomal substitution analysis of receptivity and sexual isolation in Drosophila melanogaster females. Heredity 75: 541-546.

Carracedo MC, Suarez A, Asenjo A, Casares P (1998). Genetics of hybridization between Drosophila simulans females and D. melanogaster males. Heredity 80: 17-24.

Carracedo MC, Suarez C, Casares P (2000b). Sexual isolation between Drosophila melanogaster, D. simulans and D. mauritiana: sex and species specific discrimination. Genetica 108: 155-162.

Cobb M, Ferveur JF (1996). Female mate discrimination or male responses to female stimulation? Evolution 50: 1719-1720.

Coyne JA (1989). Genetics of sexual isolation between two sibling species, Drosophila simulans and Drosophila mauritiana. Proc Natl Acad Sci USA 86: 5464-5468.

Coyne JA (1992). Genetics of sexual isolation in females of the Drosophila species complex. Genet Res 60: 25-31.

Coyne JA, Crittenden AP, Mah K (1994). Genetics of a pheromonal difference contributing to reproductive isolation in Drosophila. Science 265: 1461-1464.

Coyne JA, Oyama R (1995). Localization of pheromonal sexual dimorphism in Drosophila melanogaster and its effect on sexual isolation. Proc Natl Acad Sci USA 92: 9505-9509.
Cowling DE, Burnet B (1981). Courtship songs and genetic control of their acoustic characteristics in sibling species of the Drosophila melanogaster subgroup. Anim Behav 29: 924-935.

Das A, Mohanty S, Capy P, David JR (1995). Mating propensity of Indian Drosophila melanogaster populations with $D$. simulans: a nonadaptive latitudinal cline. Heredity 74: 562-566.

Eoff M (1977). Artificial selection in Drosophila simulans males for increased and decreased sexual isolation from $D$. melanogaster males. Am Nat 111: 259-266.

Izquierdo JI, Carracedo MC, Piñeiro R, Casares P (1992). Response to selection for increased hybridization between Drosophila melanogaster females and D. simulans males. J Hered 83: $100-104$.

Jamart JA, Carracedo MC, Casares P (1993). Sexual isolation between Drosophila melanogaster females and D. simulans males. Male mating propensities versus success in hybridization. Experientia 49: 596-598.

Kawanishi M, Watanabe TK (1981). Genes affecting courtship song and mating preference in Drosophila melanogaster, Drosophila simulans and their hybrids. Evolution 35 1128-1133.

Kyriacou CP, Hall JC (1982). The function of courtship song rhythms in Drosophila. Anim Behav 30: 794-801.

Lemeunier F, Ashburner M (1976). Studies on the evolution of the melanogaster species subgroup of the genus Drosophila (Sophophora). II. Phylogenetic relationships of six species based upon polytene chromosome banding patterns. Proc $R$ Soc Lond 193B: 137-157.

Lemeunier F, Ashburner M (1984). Relationship within the melanogaster species subgroup of the genus Drosophila (Sophophora). IV. The chromosomes of two new species. Chromosoma 89: 343-351.

Ohnishi S, Voelker RA (1979). Comparative studies of allozyme loci in Drosophila simulans and D. melanogaster. II. Gene arrangement on the third chromosome. Jpn J Genet 54: 203-209.

Savarit F, Sureau G, Cobb M, Ferveur JF (1999). Genetic elimination of known pheromones reveals the fundamental chemical bases of mating and isolation in Drosophila. Proc Natl Acad Sci USA 96: 9015-9020.

Scott D (1994). Genetic variation for female mate discrimination in Drosophila melanogaster. Evolution 48: 112-121.

Scott D, Jackson IL (1988). Interstrain comparison of malepredominant antiaphrodisiacs in Drosophila melanogaster. J Insect Physiol 34: 863-871.

Snedecor GW, Cochran WG (1967). Statistical Methods, 6th edn. Iowa State University Press: Ames.

Sokal RR, Rohlf FJ (1995). Biometry, 3rd edn. Freeman and Co: San Francisco.

Templeton AR (1982). Adaptation and integration of evolutionary forces. In: Milkman R (ed) Perspectives on Evolution. Sinauer: Sunderland, MA. pp 15-31.

Ting C-T, Takahashi A, Wu C-I (2001). Incipient speciation by sexual isolation in Drosophila: concurrent evolution at multiple loci. Proc Natl Acad Sci USA 98: 6709-6713.

Watanabe TK, Lee WH, Inoue Y, Kawanishi M (1977). Genetic variation of the hybrid crossability between Drosophila melanogaster and D. simulans. Jpn J Genet 52: 1-8. 